KYUNGPOOK Math. J. 55(2015), 13-20

http://dx.doi.org/10.5666/KMJ.2015.55.1.13

pISSN 1225-6951 eISSN 0454-8124

(c) Kyungpook Mathematical Journal

\title{
Distributivity on the Gyrovector Spaces
}

SEJONG KIM

Department of Mathematics, Chungbuk National University, Cheongju, 362-763, Korea

e-mail : skim@chungbuk.ac.kr

Abstract. As a vector space provides a fundamental tool for the study of Euclidean geometry, a gyrovector space provides an algebraic tool for the study of hyperbolic geometry. In general, the gyrovector spaces do not satisfy the distributivity with scalar multiplication. In this article, we see under what condition the distributivity with scalar multiplication is satisfied.

\section{Introduction}

In order to provide an algebraic tool to study Einstein's relativistic velocity sum, A. A. Ungar [2] has introduced a notion of gyrogroup and has developed together the study of analytic hyperbolic geometry. The gyrogroup is a most natural extension of a group into the nonassociative algebra. The associativity (and the commutativity) of group operations is salvaged in a suitably modified form, called a gyroassociativity (and a gyrocommutativity). In Section 2 we introduce a notion of (gyrocommutative) gyrogroup with its examples.

Later on it is known that gyrocommutative gyrogroups are equivalent to Bruck loops (see [1]). To elaborate a precise language, we prefix a gyro to terms that describe concepts in Euclidean geometry to mean the analogous concepts in hyperbolic geometry. The prefix gyro stems from Thomas gyration, which is the mathematical abstraction of a special relativistic effect known as Thomas precession.

Some gyrocommutative gyrogroups give rise to gyrovector spaces just as some commutative groups give rise to vector spaces. Let $(G, \oplus, \otimes)$ be a gyrovector space (see a definition of gyrovector space in Section 3), and let $x, y \in G$. In general, gyroaddition $\oplus$ does not distribute with scalar multiplication such as

$$
t \otimes(x \oplus y) \neq t \otimes x \oplus t \otimes y
$$

Received March 3, 2014; accepted July 14, 2014.

2010 Mathematics Subject Classification: 20N05, 81R05.

Key words and phrases: gyrogroup, gyrovector space, gyroautomorphism. 
We investigate in Section 4 under what condition the above distributivity is satisfied.

\section{Gyrocommutative Gyrogroups}

We start with the axioms of gyrogroup introduced by A. Ungar [2]. His axioms are reminiscent of those for a group, but gyrogroup operations are nonassociative in general.

Definition 2.1. A triple $(G, \oplus, 0)$ is a gyrogroup if the following axioms are satisfied for all $a, b, c \in G$.

(G1) $0 \oplus a=a \oplus 0=a$ (existence of identity);

(G2) $a \oplus(-a)=(-a) \oplus a=0$ (existence of inverses);

(G3) There is an automorphism gyr $[a, b]: G \rightarrow G$ for each $a, b \in G$ such that

$$
a \oplus(b \oplus c)=(a \oplus b) \oplus \operatorname{gyr}[a, b] c \text { (gyroassociativity); }
$$

(G4) $\operatorname{gyr}[0, a]=\mathrm{id}$;

(G5) $\operatorname{gyr}[a \oplus b, b]=\operatorname{gyr}[a, b]$ (loop property).

We call gyr $[a, b]$ the gyroautomorphism or Thomas gyration generated by $a$ and b. From (G2) and (G3) we have

$$
\operatorname{gyr}[a, b] c=\ominus(a \oplus b) \oplus[a \oplus(b \oplus c)]=L_{a \oplus b}^{-1} L_{a} L_{b}(c)
$$

for all $a, b, c \in G$, where $L_{x}$ denotes a left translation by $x \in G$.

Definition 2.2. A gyrogroup $(G, \oplus)$ is gyrocommutative if it satisfies

$$
a \oplus b=\operatorname{gyr}[a, b](b \oplus a) \text { (gyrocommutativity). }
$$

We consider the basic characterizations of gyrocommutative gyrogroups.

Lemma 2.1. Let $(G, \oplus)$ be a gyrogroup. Then the following are equivalent.

(1) $G$ is gyrocommutative.

(2) $G$ satisfies the automorphic inverse property; $\ominus(a \oplus b)=\ominus a \ominus b$.

(3) G satisfies the Bruck identity; $(a \oplus b) \oplus(a \oplus b)=a \oplus(b \oplus(b \oplus a))$.

Proof. The equivalence of (1) and (2) has been proved in [2, Theorem 3.2].

$(1) \Rightarrow(3)$ Assume that a gyrogroup $(G, \oplus)$ is gyrocommutative. Then for any $a, b \in G$

$$
a \oplus b=\operatorname{gyr}[a, b](b \oplus a) .
$$


From the equation (2.1) we have $\operatorname{gyr}[a, b]=L_{a \oplus b}^{-1} L_{a} L_{b}$, where $L_{x}$ is a left translation by $x$. Thus,

$$
\begin{aligned}
(a \oplus b) \oplus(a \oplus b) & =L_{a \oplus b}(a \oplus b) \\
& =L_{a \oplus b} \operatorname{gyr}[a, b](b \oplus a) \\
& =L_{a \oplus b} L_{a \oplus b}^{-1} L_{a} L_{b}(b \oplus a) \\
& =L_{a} L_{b}(b \oplus a) \\
& =a \oplus(b \oplus(b \oplus a)) .
\end{aligned}
$$

$(3) \Rightarrow(1)$ Assume that a gyrogroup $(G, \oplus)$ satisfies the Bruck identity;

$$
(a \oplus b) \oplus(a \oplus b)=a \oplus(b \oplus(b \oplus a)) .
$$

It can be replaced by

$$
L_{a \oplus b}(a \oplus b)=L_{a} L_{b}(b \oplus a) .
$$

It is known that a left translation $L_{x}$ is a bijection with inverse $L_{x}^{-1}=L_{\ominus x}$. So we have

$$
a \oplus b=L_{a \oplus b}^{-1} L_{a} L_{b}(b \oplus a) .
$$

By the equation (2.1) we obtain $a \oplus b=\operatorname{gyr}[a, b](b \oplus a)$.

We give some examples of gyrocommutative gyrogroups.

Example 2.1. Let $\mathbb{V}$ be a complex vector space equipped with inner product $\langle$,$\rangle .$ Let

$$
\mathbb{V}_{s}=\{\mathbf{v} \in \mathbb{V}:\|\mathbf{v}\|<s\}
$$

be the open $s$-ball, where $s$ is an arbitrary fixed positive constant. Define the binary operation $\oplus_{E}$ in $\mathbb{V}_{s}$ by

$$
\mathbf{u} \oplus_{E} \mathbf{v}=\frac{1}{1+\frac{\langle\mathbf{u}, \mathbf{v}\rangle}{s^{s}}}\left\{\mathbf{u}+\frac{1}{\gamma_{\mathbf{u}}} \mathbf{v}+\frac{\gamma_{\mathbf{u}}}{s^{2}\left(1+\gamma_{\mathbf{u}}\right)}\langle\mathbf{u}, \mathbf{v}\rangle \mathbf{u}\right\}
$$

for any $\mathbf{u}, \mathbf{v} \in \mathbb{V}_{s}$, where $\gamma_{\mathbf{u}}$ is the Lorentz factor such that

$$
\gamma_{\mathbf{u}}=\frac{1}{\sqrt{1-\frac{\langle\mathbf{u}, \mathbf{v}\rangle}{s^{s}}}} .
$$

The binary system $\left(\mathbb{V}_{s}, \oplus_{E}\right)$ forms a gyrocommutative gyrogroup, called the standard relativistic gyrogroup or the Einstein gyrogroup. 
Example 2.2. Let $\mathbb{P}$ be the open convex cone of all positive definite Hermitian matrices with fixed dimension. Define the binary operation $\odot$ in $\mathbb{P}$ by

$$
A \odot B=A^{1 / 2} B A^{1 / 2}
$$

for any $A, B \in \mathbb{P}$. Then the binary system $(\mathbb{P}, \odot)$ forms a gyrocommutative gyrogroup, and the gyroautomorphism generated by $A$ and $B$ is given by

$$
\operatorname{gyr}[A, B] C=U\left(A^{1 / 2}, B^{1 / 2}\right) C U\left(A^{1 / 2}, B^{1 / 2}\right)^{-1},
$$

where $U\left(A^{1 / 2}, B^{1 / 2}\right)=\left(A^{1 / 2} B A^{1 / 2}\right)^{-1 / 2} A^{1 / 2} B^{1 / 2}$ is a unitary part of the polar decomposition for $A^{1 / 2} B^{1 / 2}$ such that

$$
A^{1 / 2} B^{1 / 2}=(A \odot B)^{1 / 2} U\left(A^{1 / 2}, B^{1 / 2}\right) .
$$

\section{Gyrovector Spaces}

In this section we give a definition of (topological) gyrovector spaces, slightly different from the definition of gyrovector spaces introduced by A. Ungar in $[2$, Chapter 6].

Definition 3.1. A gyrovector space consists of a gyrocommutative gyrogroup $(G, \oplus)$ equipped with a scalar multiplication

$$
(t, x) \mapsto t \otimes x: \mathbb{R} \times G \rightarrow G
$$

satisfying the following: for any $s, t \in \mathbb{R}$ and $a, b, x \in G$

(S1) $1 \otimes x=x, 0 \otimes x=0=t \otimes 0$, and $(-1) \otimes x=\ominus x$;

(S2) $(s+t) \otimes x=s \otimes x \oplus t \otimes x$;

(S3) $s \otimes(t \otimes x)=(s t) \otimes x$;

(S4) $\operatorname{gyr}[a, b](t \otimes x)=t \otimes \operatorname{gyr}[a, b] x$.

Definition 3.2. A topological gyrovector space is a gyrovector space $(G, \oplus, \otimes)$ equipped with Hausdorff topology such that both $\oplus: G \times G \rightarrow G$ and $\otimes: \mathbb{R} \times G \rightarrow G$ are continuous.

Example 3.1. In the open $s$-ball $\mathbb{V}_{s}$ of a complex vector space $\mathbb{V}$ equipped with inner product $\langle$,$\rangle we define$

$$
t \otimes \mathbf{v}= \begin{cases}s \tanh \left(t \tanh ^{-1} \frac{\|\mathbf{v}\|}{s}\right) \frac{\mathbf{v}}{\|\mathbf{v}\|}, & \mathbf{v} \neq \mathbf{0} ; \\ \mathbf{0}, & \mathbf{v}=\mathbf{0} .\end{cases}
$$

for any $t \in \mathbb{R}$. Then $\left(\mathbb{V}_{s}, \oplus_{E}, \otimes\right)$ is a gyrovector space, called the Einstein gyrovector space. 
Example 3.2. On the open convex cone $\mathbb{P}$ of positive definite Hermitian matrices, we define

$$
t \star A=A^{t}
$$

for any $t \in \mathbb{R}$ and $A \in \mathbb{P}$. Then $(\mathbb{P}, \odot, \star)$ is a gyrovector space. Indeed, let us check (S4). For any $A, B, X \in \mathbb{P}$

$$
\begin{aligned}
\operatorname{gyr}[A, B](t \otimes X) & =U\left(A^{1 / 2}, B^{1 / 2}\right) X^{t} U\left(A^{1 / 2}, B^{1 / 2}\right)^{-1} \\
& =U\left(A^{1 / 2}, B^{1 / 2}\right) \exp (t \log X) U\left(A^{1 / 2}, B^{1 / 2}\right)^{-1} \\
& =\exp \left[t \log U\left(A^{1 / 2}, B^{1 / 2}\right) X U\left(A^{1 / 2}, B^{1 / 2}\right)^{-1}\right] \\
& =\left[U\left(A^{1 / 2}, B^{1 / 2}\right) X U\left(A^{1 / 2}, B^{1 / 2}\right)^{-1}\right]^{t}=t \otimes \operatorname{gyr}[A, B] X .
\end{aligned}
$$

\section{Distributivity}

Let $(G, \oplus, \otimes)$ be a topological gyrovector space, and let $x, y \in G$. In general, gyroaddition $\oplus$ does not distribute with scalar multiplication such as

$$
t \otimes(x \oplus y) \neq t \otimes x \oplus t \otimes y .
$$

We here investigate under what condition the above distributivity is satisfied.

Lemma 4.1. For any natural numbers $m$ and $n$ the following are equivalent.

(i) $\operatorname{gyr}[x, y]=I$.

(ii) $\operatorname{gyr}[m \otimes x, n \otimes y]=I$.

Proof. It is enough to show (i) $\Rightarrow$ (ii), and we use the induction for $n$ and for $m$. We assume that $\operatorname{gyr}[x, y]=I$, that is, $x \oplus y=y \oplus x$ by gyrocommutativity.

Suppose that $\operatorname{gyr}[x,(n-1) \otimes y]=I$, or $x \oplus(n-1) \otimes y=(n-1) \otimes y \oplus x$. Then by gyroassociativity

$$
\begin{aligned}
x \oplus n \otimes y & =x \oplus\{y \oplus(n-1) \otimes y\} \\
& =(x \oplus y) \oplus(n-1) \otimes y \\
& =(y \oplus x) \oplus(n-1) \otimes y \\
& =y \oplus\{x \oplus(n-1) \otimes y\} \\
& =y \oplus\{(n-1) \otimes y \oplus x\} \\
& =n \otimes y \oplus x .
\end{aligned}
$$

We now use the induction for $m$. From the preceding we have shown $\operatorname{gyr}[x, n \otimes y]=I$ when $m=1$. Suppose that $\operatorname{gyr}[(m-1) \otimes x, n \otimes y]=I$, or $(m-1) \otimes x \oplus n \otimes y=$ 
$n \otimes y \oplus(m-1) \otimes x$. Then by gyroassociativity

$$
\begin{aligned}
m \otimes x \oplus n \otimes y & =\{x \oplus(m-1) \otimes x\} \oplus n \otimes y \\
& =x \oplus\{(m-1) \otimes x \oplus n \otimes y\} \\
& =x \oplus\{n \otimes y \oplus(m-1) \otimes x\} \\
& =\{x \oplus n \otimes y\} \oplus \operatorname{gyr}[x, n \otimes y](m-1) \otimes x \\
& =\{n \otimes y \oplus x\} \oplus(m-1) \otimes x \\
& =n \otimes y \oplus m \otimes x .
\end{aligned}
$$

Remark 4.1. On a gyrovector space $(G, \oplus, \otimes)$ each element $x$ has a unique $n$th root in $G$, denoted by $\frac{1}{n} \otimes x$. From Lemma 4.1 we have an alternative version of equivalence.

(i) $\operatorname{gyr}[x, y]=I$.

(ii) $\operatorname{gyr}\left[\frac{1}{m} \otimes x, \frac{1}{n} \otimes y\right]=I$.

Proposition 4.1. For any integer $n$ the following are equivalent.

(i) $\operatorname{gyr}[x, y]=I$.

(ii) $n \otimes(x \oplus y)=n \otimes x \oplus n \otimes y$.

Proof. First, we show that (i) $\Rightarrow$ (ii) by induction for natural number $n$. Obviously (ii) holds for $n=0$ and $n=1$. We assume that (ii) holds for $n=k-1$. Then

$$
\begin{aligned}
k \otimes(x \oplus y) & =(x \oplus y) \oplus(k-1) \otimes(x \oplus y) \\
& =(x \oplus y) \oplus(k-1) \otimes(y \oplus x) \\
& =(x \oplus y) \oplus\{(k-1) \otimes y \oplus(k-1) \otimes x\} \\
& =x \oplus[y \oplus\{(k-1) \otimes y \oplus(k-1) \otimes x\}] \\
& =x \oplus\{k \otimes y \oplus(k-1) \otimes x\} \\
& =x \oplus \operatorname{gyr}[k \otimes y,(k-1) \otimes x]\{(k-1) \otimes x \oplus k \otimes y\} \\
& =x \oplus\{(k-1) \otimes x \oplus k \otimes y\} \\
& =k \otimes x \oplus k \otimes y .
\end{aligned}
$$

The second equality follows from (i) $\operatorname{gyr}[x, y]=I$, the third follows from the assumption, the fourth, the fifth, and the last follow from the gyroassociativity, the sixth follows from the gyrocommutativity, and the seventh follows from Lemma 4.1.

Secondly, we show that (i) $\Rightarrow$ (ii) for negative integer $n$. We take $n=-p$ for some positive integer $p$. Then

$$
\begin{aligned}
n \otimes(x \oplus y) & =p \otimes\{(-1) \otimes(x \oplus y)\} \\
& =p \otimes\{(-x) \oplus(-y)\} \\
& =p \otimes(-x) \oplus p \otimes(-y) \\
& =n \otimes x \oplus n \otimes y .
\end{aligned}
$$


The second equality follows from the automorphic inverse property, and the third follows from (ii) for natural number $p$ and $\operatorname{gyr}[-x,-y]=\operatorname{gyr}[x, y]=I$.

Finally, we show that (ii) $\Rightarrow$ (i). For any $x$ and $\frac{1}{2} \otimes y$, (ii) gives us

$$
2 \otimes\left(x \oplus \frac{1}{2} \otimes y\right)=2 \otimes x \oplus y .
$$

By Bruck identity and the gyroassociativity we have

$$
\begin{aligned}
2 \otimes\left(x \oplus \frac{1}{2} \otimes y\right) & =x \oplus\left\{\frac{1}{2} \otimes y \oplus\left(\frac{1}{2} \otimes y \oplus x\right)\right\} \\
& =x \oplus(y \oplus x) .
\end{aligned}
$$

Then $x \oplus(y \oplus x)=2 \otimes x \oplus y=x \oplus(x \oplus y)$. By the left cancellation, $y \oplus x=x \oplus y$, and hence, we obtain (i) $\operatorname{gyr}[x, y]=I$.

Remark 4.2. From Remark 4.1 and Proposition 4.1 we obtain the equivalence for any nonzero integer $n$ :

(i) $\operatorname{gyr}[x, y]=I$,

(ii) $\frac{1}{n} \otimes(x \oplus y)=\frac{1}{n} \otimes x \oplus \frac{1}{n} \otimes y$.

We finally see that the property $\operatorname{gyr}[x, y]=I$ is a sufficient and necessary condition to satisfy

$$
t \otimes(x \oplus y)=t \otimes x \oplus t \otimes y
$$

for any real number $t$.

Theorem 4.1. Let $(G, \oplus, \otimes)$ be a topological gyrovector space, and let $x, y \in G$. The following are equivalent for any real number $t$.

(i) $\operatorname{gyr}[x, y]=I$.

(ii) $t \otimes(x \oplus y)=t \otimes x \oplus t \otimes y$.

Proof. It is enough to show that (i) $\Rightarrow$ (ii) for any dyadic rational number $t=\frac{m}{2^{n}}$, where $n$ is a natural number and $m$ is an integer, since the set of all dyadic rational numbers is dense in the set of real numbers and the scalar multiplication $\otimes$ is continuous.

$$
\begin{aligned}
\frac{m}{2^{n}} \otimes(x \oplus y) & =m \otimes\left\{\frac{1}{2^{n}} \otimes(x \oplus y)\right\} \\
& =m \otimes\left(\frac{1}{2^{n}} \otimes x \oplus \frac{1}{2^{n}} \otimes y\right) \\
& =\frac{m}{2^{n}} \otimes x \oplus \frac{m}{2^{n}} \otimes y .
\end{aligned}
$$


The second equality follows from Remark 4.1, and the third follows from Proposition 4.1.

\section{References}

[1] L. V. Sabinin, L. L. Sabinin, and L. V. Sbitneva, On the notion of a gyrogroup, Aequ. Math., 56(1998), 11-17.

[2] A. A. Ungar, Analytic hyperbolic geometry and Albert Einstein's special theory of relativity, World Scientific Press, 2008. 